\title{
LA FIGURA DELLA MADRE NEL ROMANZO “DIECI GOCCE" DI GIORGIO TODDE
}

\author{
THE ROLE OF THE MOTHER IN THE NOVEL “DIECI GOCCE” BY GIORGIO \\ TODDE
}

Irene Scampuddu

Universidad de Salamanca

\section{Riasunto:}

Giorgio Todde medico e scrittore cagliaritano, ci porta con il suo romanzo Dieci gocce ad uno studio sulle manie e le follie umane riunite nelle complesse personalità di Mario e di sua madre. Quest'ultima è l'unica che lo compatisce e non gli da tregua nel provare a portarlo verso atteggiamenti positivi, e Todde la descrive utilizzando un forte sarcasmo, durezza e insensibilità.

\section{Parole Chiave:}

Giorgio Todde, letteratura sarda, Dieci gocce, dura madre.

\section{Abstract:}

Giorgio Todde, a physician and writer from Cagliari, in his novel Dieci Gocce, takes us throughout a study of human manias and craziness as seen in the complex personalities of Mario and his mother. The latter is the only one that shows him compassion and doesn't give him a break, Todde describes her utilizing a great deal of sarcasm, toughness and insensitivity.

\section{KEY WORD:}

Giorgio Todde, Sardinian litterature, Dieci Gocce, strong mother. 
Giorgio Todde, cagliaritano, scrittore e medico, è considerato insieme a Marcello Fois ${ }^{1}$, uno dei più grandi scrittori sardi contemporanei.

Fondatore, insieme a Michela Murgia, Marcello Fois e Giulio Angioi, dell'importante Festival di Gavoi e massimo esponente della nuova letteratura sarda.

Giorgio Todde si è reso noto al grande pubblico però, per aver inserito all'interno dei suoi romanzi gialli, la figura di Efisio Marini.

Sono cinque i romanzi noir dedicati appunto al medico-imbalsamatore Efisio Marini un personaggio realmente esistito, e una narrazione la sua dedicata alla storia di un'angoscia durata tutta la vita è tutto fuorché una vicenda dal taglio diagnostico e quindi consolatorio.

Come lo stesso autore, è un medico in carne e ossa. Fu un importante e incompreso scienziato di Cagliari che nella seconda meta del secolo XIX ottenne un metodo segreto

e efficace per la pietrificazione dei cadaveri. Todde si distingue rispetto a molti altri autori proprio per aver recuperato questo insolito e per certi versi marginale personaggio per convertirlo in un detective di una serie di novelle che oltretutto descrivono epoche differenti della sua vita. Marini sarà quindi il protagonista dei romanzi gialli di Todde: Lo stato delle anime (2001), Paura e carne (Frassinelli, 2003) L'occhiata letale (2004), E quale amore non cambia (Frassinelli, 2005) e L'estremo delle cose (2007), dove le inchieste condotte da Efisio Marini, si chiudono, almeno sin ora, con la morte del detective-mumificatore.

Todde non difende un progetto d'identità dichiarato anzi, racconta di una Sardegna metropolitana, mediterranea, distante dalle logiche barbaricine e aperta ad altre filosofie di vita, quindi una Sardegna ben distante da quella tradizionale.

Mentre le altre sue opere sono ascrivibili al genere giallo o al noir metafisico, il romanzo Dieci gocce di cui parlerò in questa sede, è il «grottesco e psicologicamente penetrante ritratto di una generazione ansiosa e incerta» (Panella 2011), e può essere considerato uno studio sulle manie e le follie umane ${ }^{2}$, riunite nella complessa personalità del protagonista, Mario.

Questo romanzo è un viaggio in cui le tortuosità di corpo e psiche si fondono, dove sublime e animalesco convivono, un luogo che possiamo chiamare "pazzia". Un ingegno folle attraversa la scia criminale intorno alla quale si muovono gli eccentrici personaggi della storia.

1. Per uno studio approfondito sulla personalità letteraria di Marcello Fois, si consiglia la lettura dell monografia a lui dedicata da Margherita Marras (2009).

2 All'opera di Giorgio Todde sono dedicati diversi studi di Giovanna Caltagirone.
Più intensa e ricca appare la scrittura di questo libro rispetto alle prove precedenti, osservando le descrizioni, l'ironia pervasiva, le riflessioni che s'addensano qua e là sui temi più disparati, lasciando affiorare weltanschauung e probabili ascendenze letterarie.

Un immagine che richiama l'origine orfica, sciamanica della poesia, e dei primi cantori, ma che richiama pure il rapporto morte-arte presente nell'opera di Thomas

Mann. In certe descrizioni invece, si avvertono echi dell'opera di Sergio Atzeni, scrittore cagliaritano, conoscitore della realtà isolana. Quando invece la visione si fa orrida, la realtà si deforma sinistramente, sembra di ritrovare certe atmosfere descritte da Friedrich Durrenmatt.

Il romanzo si focalizza soprattutto non solo nella figura di Mario, ma soprattutto in quella di sua madre, una donna spesso crudele e quasi sempre una figura decisiva nella vita del figlio.

La storia è ambientata in una città cui non possiamo dare un nome preciso, ma dietro la quale possiamo intuire il profilo di Cagliari; si assiste ad un delitto, ma non possiamo parlare di giallo, poiché non esiste alcun mistero intorno al crimine, e inoltre l'attenzione del lettore è completamente assorbita dalle ansie dei personaggi, il cui principale obiettivo è l'incorruttibilità del proprio corpo.

Al centro della storia c'è Mario, affetto da manie compulsive che ha sempre sofferto di ansia, di svenimenti, di trasalimenti legati alla salute e alla necessità $\mathrm{d}$ vivere comunque una vita normale, come tutti fanno nel corso del tempo che passa. Archivista modello, creatore poetico di altrimenti gelide pratiche burocratiche che cerca di arricchire di umanità, Mario ha bisogno di una vita inquadrata entro perimetri ben definiti, potremmo dire quasi ferrei..

Mario vive ancora con la madre, nonostante abbia superato abbondantemente la trentina. Le sue ossessioni lo spingono alla continua ricerca della pulizia e dell'ordine perfetti, ma anche dei minimi segni del degrado fisico. Todde ci porta nei meandri della testa dell'uomo, nei suoi labirintici ragionamenti, a cui solo le dieci gocce di calmante, che danno il titolo all'opera, possono imporre una fine momentanea.

Le donne che animano le sue giornate (sua madre, sua moglie e le due amanti) provano ad alleviare i suoi mali psicofisici, senza riuscirci, e benché Mario tenti d isolarsi dal mondo, per non subire il peso delle angosce o delle gioie altrui, le figure femminili del romanzo non lo lasciano mai solo e giustificano le sue manie e ossessioni.

L'unica che non lo compatisce e non gli dà tregua nel provare a portarlo verso atteggiamenti più positivi, è sua madre:

- Credi che non le conosco le tue manie? Che non conosco l'ordine da matto delle tue cose? Se qualcuno te le cambia anche un pochino, è vero o no che ti vengono 
la nausea e i bruciori di stomaco? Quante volte hai vomitato il caffelatte perche prima non ti eri lavato i denti?- E perché non mi ero lavato i denti? Dillo, su,dillo.

- Perché nel bagno c'ero io, d'accordo. Ma uno normale va nell'altro bagno. Tu no.

Tu vomiti! Vomitavi anche dopo il biberon. (Todde 2010, p. 17)

Seguendo il punto di vista di Mario, il personaggio della madre viene descritto e trattato unicamente nei suoi aspetti negativi. Lei rappresenta la nemesi del figlio, una sorta di personificazione della giustizia in contrasto col pessimismo dell'uomo. Lei vuole approfittare della vita che le resta, nonostante l'età e un ictus che ha sformato il suo corpo. Rimasta sola dopo la morte del padre, ma ormai insofferente alla presenza costante del figlio in casa, vorrebbe che il suo unico e immobile rampollo si sistemasse e la lasciasse vivere fino in fondo la sua relazione con il pur anziano dottor Cosimo, da molto tempo medico di famiglia. E' quest'ultimo a prescrivere a Mario le "dieci gocce" di tranquillanti che danno il titolo alla narrazione di Todde. Ma le gocce non sempre bastano e anche una vita che eviti ogni compromissione con il mondo esterno (esemplare è l'abitudine di Mario di andare al lavoro rigorosamente chiuso nella sua automobile con i finestrini praticamente sigillati) non è sufficiente a preservare dall'impatto spaventoso con una vita odiata e temuta.

Un giorno, però, una ragazza dal "collo indifeso" e dalle belle ginocchia unite gli passa a fianco anch'essa difesa da un'automobile ben chiusa. Il collo e le ginocchia d quella donna lo attirano ma Mario cerca di evitare la tentazione. Aprirsi e proiettarsi all'esterno gli sembra troppo pericoloso.

Per sfuggire alla tentazione della ragazza, Mario inizia una relazione con Tosca, un travestito, del quale si innamora anche se poi si trasformerà, nonostante l'investimento affettivo dell'uomo, in una relazione di tipo mercenario. Questa condizione sarà mitigata, in seguito, dall' affetto sincero dell'uomo che si prostituisce ma che, però, nonostante senta qualcosa per lui, non vuole avere una relazione stabile con l'impiegato. Mario, in seguito a questa delusione, finirà per prendere una decisione radicale presentandosi, spinto dalla madre e rinforzato dalle gocce prese precedentemente davanti al portone del palazzone di mattoni dove si trova l'ufficio in cui lavora la ragazza e le chiede di salire in macchina con lui. Dopo averla corteggiata in maniera incerta e infantile, Alda (è questo il nome della donna) diverrà prima la sua amante e poi sua moglie, ma il matrimonio (e poi la paternità) non guariscono l'uomo; le gocce restano in realtà l'unico rimedio sovrano per l'ansia e l'angoscia di vivere. E' vero che Alda diventerà presto fondamentale per Mario e la sua presenza costituirà per lui una sorta di ancora di salvezza, energia forte e quasi inestinguibile. La figura di Alda quale sua salvatrice sembra aver rassicurato Mario.
Successivamente però la morte di Cosimo che era divenuto il convivente ufficiale della madre dopo il matrimonio del figlio e un ictus che colpisce la donna, riaprono i conti con la morte. Infine, anche lui subirà repentinamente il colpo di un infarto.

Mario nell'ospedale in cui viene ricoverato, conosce Mariannina, un'infermiera di straordinaria vitalità che si impadronisce di lui e ne diviene l'amante. Di fronte al consolidarsi di questo nuovo rapporto, Alda sembra rimanere inerte e accettare il fatto compiuto, come pure farà la madre ormai invalida e costretta all'uso di bastoni e deambulatori per spostarsi. Ma c'è di più: Mariannina conduce Mario a una riunione del Consiglio di circoscrizione e lo spinge a parlare in pubblico.

Il risultato ottenuto è straordinario: la sua idea che ciascuno abbia bisogno di protezione e che l'instaurazione generalizzata di essa, la sua istituzionalizzazione, condurrebbe gli uomini a ridurre le loro paure e le loro angosce conquista gli elettori che lo votano in gran numero. Le tesi di Mario inoltre, attirano l'attenzione dell'Assessore agli Affari Sociali, Guido Langelico, che incarica l'ormai ex-impiegato di creare un apposito Ufficio Protezione in cui ricevere, confortare e curare tutti quelli che hanno paura di ciò che non si può curare o sopperire direttamente. Il successo dell'iniziativa è enorme e Mario riceve perfino l'appoggio del priore del convento più potente della zona (al quale non ha neppure nascosto il suo sostanziale ateismo).

Purtroppo però, in seguito a un secondo infarto e dopo aver ripreso le sue attività come direttore dell'Ufficio Protezione, le due donne di Mario restano incinte quas contemporáneamente, saranno per l'uomo il secondo figlio da Alda e il primo avuto da Mariannina.

Mario, nonostante la situazione in cui si trova, ormai solidamente insediato nella sua nuova attività, conosce Giulia, una donna instabile e malata e se ne innamora suscitando il forte risentimento di Alda che più volte cercherà di ucciderlo.

Ma Mario continua ad esserne attirato finché per riuscire a staccarsene decide lui di ucciderla ${ }^{3}$ : compra un coltello "corrusco" e la fa morire. A questo punto è pronto anche lui per la fine. Le gocce non bastano più:

Le gocce diventano subito me stesso. Buttare giu una pastiglia è un'azione che chiunque puo fare, basta avere la bocca. Le gocce no, le gocce richiedono intelligenza, esperienza, perfino una tattica e tanta sensibilita. Bisogna conoscers bene, sapere di quanto c'e bisogno e sapere cosa si vuole. Mi sono convinto che il gesto delle gocce sulla lingua e quello del coltello possiedono proprio la stessa armonia. Sono passate due settimane. Un sole incessante, gillognolo come me, un vento umido che ha infradiciato tutto. Guglia, ormai, si sarà ridotta a chissa che cosa. La vorrei vedere. Sono sicuro che è composta, però, e che ha mantenuto l'ordine (pp. 276-277)

3 Uccidere per amore, come i "veri" amanti descritti nella Ballata del carcere di Reading di Oscar Wilde.

(a) 2017 
Si assiste, nel corso della lettura, allo sgretolarsi di quello che appare,all'inizio, come un muro, contro cui le paure e le obiezioni di Mario non possono nulla, e contro cui nemmeno la natura sembra poter niente: dapprima, si intuisce una condizione di schiavitù psicologica di Mario rispetto all'anziana donna, che si mostra insensibile ai suoi dolori e sembra passi il suo tempo a denigrarlo. In realtà, più la storia evolve, più si comprende che lo schema è capovolto, ovvero è la vecchia ad essere succube delle manie del figlio.

La freddezza, ostentata in maniera sarcastica, non è altro che il frutto dell'istinto di sopravvivenza, l'unico modo per contrastare l'ossessività morbosa del figlio, in preda a continue crisi di panico, che fa della sua ipocondria un prisma di lettura del mondo che lo circonda.

Ritratta come una sorta di cariatide, "carnivora per natura, onnivora per necessità» (Todde 2010, p. 119), che lotta contro la legge di gravità, che fa crollare pezzo per pezzo tutto il suo fisico, la madre è descritta in maniera caricaturale, mentre tenta di aggiustarsi col rossetto la bocca colpita dall'ictus o si sistema la dentiera.

Prigioniera in un corpo ormai deforme, cerca di spronare in tutti i modi il figlio a non rincorrere in modo spasmodico una vecchiaia tranquillizzante, preludio di una fine che lui teme ed agogna allo stesso tempo.

Il rapporto tra i due si snoda attraverso dei siparietti comici, che costituiscono punti di luce in un romanzo decisamente scuro e deprimente:

\section{Mamma, mamma...}

Non chiamarmi mamma due volte. Sembri un ritardato. Chiamami una sola volta

e io ri-spondo. [...]

- Mamma.

- Mamma puzza d'ospedale, questo vuoi dirmi? Mamma ha un cattivo odore anche se si

spruzza profumo ad ogni angolo del corpo.(Todde 2010, p. 195)

L'atteggiamento sprezzante della madre nei confronti di Mario permette di individuare in lei l'apoteosi del modello letterario di dura madre nei romanzi di Giorgio Todde.

La sua durezza è un insieme di sarcasmo, distanza e insensibilità, che, però, sono il risultato delle ossessioni del figlio, e quindi una reazione a stimoli esterni e non la manifestazione di una crudeltà innata.

A differenza delle madri descritte in Paura e carne e in E quale amor non cambia, quella di Dieci gocce mostra una forma di amore materno assopito, esasperato forse, ma non assente.

L'analisi condotta su Paura e carne e Dieci gocce sottolinea, dunque, un elemento comune ai due romanzi: la presenza di una figura materna distaccata, che non manifesta le classiche caratteristiche di una madre, ovvero la dolcezza, l'amore incondizionato e gratuito e un senso di totale altruismo verso la propria progenie. Al contrario, emerge un preciso calcolo sia nell'atto della procreazione che in quello della cura della famiglia, un sostanziale egoismo che si esprime nell'inseguire unicamente il proprio benessere e nel rifiutarsi di reprimere la propria personalità a beneficio dei figli

Voi lo volete intatto il vostro corpo, è naturale perché il corpo vuole questo, a questo tende. Perciò si è richiuso subito dietro Giovanni dopo che l'avete partorito. Chiuso per sempre. Voi vi siete riprodotta non perché i mammiferi sono spint a riprodursi ma perché dovevate completare un corredo di cose utili. E questo corredo poi è aumentato. Avete imparato gli affari, le economie, dite voi. (Todde 2003, p. 252)

Una visione utilitaristica della maternità, dunque, frutto di un puro calcolo. Nella Sardegna di Todde, regna un estremo individualismo, tipico, forse, della vita cittadina che l'autore racconta nei suoi romanzi; principalmente esistono solo gli interessi privati dei singoli, con buona pace degli eventuali legami di parentela.

"Sa femina" , che in questo romanzo assume, in maniera spontanea e silenziosa, un ruolo superiore a quello maschile che fa un passo indietro, ma non perchè considerato meno importante, ma perchè alla luce della ribaltà in questo sistema di matriarcato ${ }^{6}$ il ruolo della donna è dominante.

Dessì pensa alle donne sarde come tante Penelopi senza Ulisse (Dessì, 1956). Per secoli e secoli sono state al telaio tessendo quei tappeti di cui, gli uomini, sono tanto fieri, ma con l'unica differenza che l'uomo sardo non stava navigando in mari lontani, si trovava al massimo a un kilometro di distanza a lavorare la terra o a controllare il bestiame perdendosi così quei lavori preziosi.

Sono dure forse le parole di Dessì però, è anche che vero, che era difficile adeguarsi a donne come queste. Donne così fedeli, costanti, coraggiose e così resistenti alla solitudine.

\footnotetext{
Per uno studio sulle madri nella letteratura sarda contemporanea, si veda l'opera di Tania Baumann Donna Isola

nella quale la critica analizza le figure materne nelle storie di Grazia Deledda, Giuseppe Dessì, Salvatore Satta, Maria

Giacobbe, Salvatore Mannuzzu e Marcello Fois. Per un'analisi specifica della figura della madre ne Il giorno del giudizio, l'opera più importante di Salvatore Satta, si consiglia la lettura del saggio di Andrea Cannas, «La madre ne Il giorno del giudizio, ovvero Donna Vincenza nel labirinto».

5 Per uno studio dettagliato consultare "La mia Sardegna arcaica”, in Gazzetta di Parma, 22 luglio 2010, p. 5.

6 Importante la lectura del saggio di Johann J. Bachofen (2004).
} 
Questa era la donna sarda, soprattutto la donna delle zone interne della Sardegna: forte e indipendente ${ }^{7}$.

La madre, nel romanzo di Todde, vive nel proprio egoismo e di questo si nutre, e la durezza non è altro che un'arma di appropriazione e di difesa dei propri spazi vitali.

Dignità e sicurezza sono i valori che la caratterizzano e derivano dall'osservanza di regole e riti codificati, ma anche dagli ideali che ha costruito ed elaborato nel corso degli anni, nonostante le difficoltà della vita.

Per questo Todde la descrive come un personaggio che ha maturato un carattere così forte, così come ha forgiato la sua dignità e la sua ingegnosità.

Simile a questa madre, quella invece descritta da Marcello Fois nel suo romanzo

appunto "Dura Madre", Mariangela Mulas, che viene inicialmente descritta come una donna anziana molto malata affetta da una demenza senile per la quale non si rende nemmeno conto dei lutti che colpiscono la sua famiglia e la sua casa.

Peraltro, aldilà della lettura più immediata del titolo del romanzo "Dura Madre" come riferimento alla protagonista Mariangela Mulas, e oltre ad un accenno alla membrana del cervello, la dura madre appunto, che richiama alla mente l'idea di protezione, in fondo non si deve escludere un'interpretazione metaforica, che vedrebbe la Sardegna dietro il titolo di «dura madre».

Il discorso da fare per Giorgio Todde è diverso. Ben distante dai lacci della sardità tradizionale, imprescindibili per molti autori contemporanei, egli non difende un progetto identitario dichiarato come quello foisiano, anzi, racconta di una Sardegna

metropolitana, mediterranea, distante dalle logiche barbaricine e aperta ad altre filosofie di vita.

Bisogna anche dire che le madri descritte da Marcello Fois sono parte attiva della famiglia, agiscono nell'ombra perché i figli ottengano rispetto e giustizia o sono garant dell'onore familiare, mentre quelle di Todde per quanto dure, non fanno altro che difendere i propri spazi.

\section{RIFERIMENTI BIBLIOGRAFICI}

Amendola, Amalia M.., L'isola che sorprende: "La narrativa sarda in italiano" (1974-2006), Cagliari, CUEC, 2000

7 Alcune riflessioni sulla figura della madre si possono trovare nel romanzo di Michela Murgia, presen in «L 'Accabadora di Michela Murgia, I'ultima madre», di Monica Acito. L'importanza che la scrittrice attribuisce al ruoolo materno nella società sarda è evidele in un brve testo, Ali traccia un percorso sentito e commovente della figura della donna sarda lungo i secoli, con tutto l'orgoglio,

e le ferite portate dentro dalle madri della sua terra.
Anon, "Paura e carne" [online]. Recensione. Italialibri.net, 5 agosto 2003. Internet. 0803-2017.<http://www.italialibri.net/opere/pauraecarne.html>.

Anon. "Il coraggio dell'indipendenza" [online]. D di Repubblica, 30 luglio 2010. Internet. 02-03-2017.<http://www.michelamurgia.com/sardegna/indipendenza/473-il coraggiodellindipendenza $>$.

Baumann, T., Donna Isol", Cagliari, CUEC, 2007.

Caltagirone, G., "La sfida alla cancellazione della memoria nell'opera di Giorgio Todde", en Insularità immagini e rappresentazioni nella narrativa sarda del Novecento, Roma, Bulzoni Editore, vol. 1, 2011, pp. 139-159.

Caltagirone, G.,"Alla ricerca dell'insularità: la scienza, la letteratura, gli indizi, nell'opera di Giorgio Todde", en Insularità e cultura mediterranea nella lingua e nella letteratura italiane, Firenze, Franco Cesati, 2012, pp. 447-455.

Cannas, A., "La madre ne Il giorno del giudizio, ovvero Donna Vincenza nel laberinto”, La figure de la mère dans la littérature contemporaine. E-talis,1. Internet. 10-03-2017.<http:// www.e-talis.com/n1_la_mere/2_cannas.pdf, 2013>.

Dessì, G., Scoperta della Sardegna, Milano, Edizioni Il Polifilo, 1956.

De Luca, E., In nome della madre, Milano, Feltrinelli, 2006.

Fois, M., Meglio morti, Torino, Einaudi, 2000.

Fois, M., Dura Madre, Torino, Einaudi, 2001.

Panella, Giuseppe, "Ansia, esistenza sovrana. Giorgio Todde, Dieci gocce", La poesia e lo spirito, 27 maggio 2011. Internet. 06-03-2017. <https://lapoesiaelospirito.wordpress com/2011/05/27/storia-contemporanea-n-74-ansia-esistenza-sovrana-giorgiotodde-dieci-gocce/ >

Todde G., Dieci Gocce, Milano, Frassinelli, 2009.

Todde, G., Lo stato delle anime, Nuoro, Il Maestrale, 2001.

Todde G., E qual amor non cambia, Nuoro, Il Maestrale, 2005.

Todde, Giorgio, Paura e carne. Nuoro-Milano, Il Maestrale, Frassinelli, 2003. 